\title{
Aortentelefon: the Berlin project aiming for shorter response times and sharper diagnostic accuracy in acute type A aortic dissection
}

\author{
Silke Zschaler ${ }^{1}$, Gerard Schmidt ${ }^{2}$, Stephan Dominik Kurz ${ }^{1}$ \\ ${ }^{1}$ Deutsches Herzzentrum Berlin, Institute for Anesthesiology, Berlin, Germany; ${ }^{2}$ Deutsches Herzzentrum Berlin, Department of Cardiothoracic and \\ Vascular Surgery, Berlin, Germany \\ Correspondence to: Dr. Stephan Dominik Kurz, MPH. Deutsches Herzzentrum Berlin, Institute for Anesthesiology, Augustenburger Platz 1, 13353 \\ Berlin, Germany. Email: skurz@dhzb.de.
}

Submitted Jul 29, 2018. Accepted for publication Sep 20, 2018.

doi: $10.21037 /$ cdt.2018.09.19

View this article at: http://dx.doi.org/10.21037/cdt.2018.09.19

Acute type A aortic dissection (ATAAD) is an emergency requiring immediate transfer to a specialized cardiac center. It requires excellent cooperation between all parties involved, with the inclusion of imaging and information technology services (1-4).

We are striving to decrease the response time of all health care providers involved during the admittance process of patients with suspected ATAAD. One of the main influencing factors on the time between the onset of pain and the surgery ("pain-to-cut-time", PCT) is the time it takes for health care providers to classify their patients' symptoms as suspected ATAAD (5).

In the region of Berlin-Brandenburg, more than $60 \%$ of all ATAAD patients receive their first medical attention by emergency medical services (EMS) (5). It is of particular importance that as soon as EMS suspects an ATAAD it must be clearly communicated and included in further differential diagnostic considerations.

\section{The aortic emergency hotline ("Aortentelefon")}

Diagnosing an ATAAD is a challenging task. Statistics show only 21 percent of the patients with a confirmed ATAAD were initially diagnosed accurately (3). For the region of Berlin-Brandenburg, there is 11.9 incidents per year, per 100,000 inhabitants which translates to 725 new cases of ATAAD per year (6).

The Deutsches Herzzentrum Berlin (DHZB) established the Aortentelefon to improve pre-hospital management and response times. Under the direction of the Institute of Anesthesiology, the DHZB provides twenty-four-seven support for optimizing pre-hospital patient management either via telephone or via the homepage of the DHZB (https://www.dhzb.de/de/service/im_notfall/). Health care providers who wish to admit their patients register them at the dispatch center for police, fire and ambulance services who will organize intensive care transport for the patient and file an electronic patient transfer record via the homepage of the DHZB.

In the Berlin-Brandenburg region, the PCT used to be eight hours but after establishing the Aortentelefon, the "pain-to-cut-time" can be reduced by up to $40 \%$ (5-7).

Unpublished data of the DHZB which has been gathered since 2006, suggests that encouraging open communication between initial health care providers and the patient's receiving party greatly assisted in reducing the PCT. While preparing this unpublished data for analysis, it was very apparent you should not waste crucial time weighing all the pros and cons until you think you have found enough evidence for a diagnosis. Any suspected ATAAD should be communicated as early as possible, even if the probability seems minute (5). Electrocardiographically gated computed tomography is considered the gold standard in diagnostics of an ATAAD (4).

\section{Aortic dissection detection risk score (ADD-RS)}

In pre-hospital settings, the ADD-RS has proven a highly sensitive clinical tool for assessing patients with suspected ATAAD (1,6-8). It covers three different aspects that help 
determine an elevated risk: conditions, pain and exam features.

The anamnestic part of the ADD-RS covers aspects in medical history that were associated with a suspected ATAAD, like a family history of aortic diseases, Marfan syndrome, known thoracic aortic aneurysm, aortic valve disease, or recent aortic manipulation.

The pathognomonic pain feature in ATAAD is described as ripping or tearing chest pain, with an abrupt onset at maximum intensity and a widely radiating anterior-posterior pattern. The ADD-RS considers pain to the chest, back or abdomen, an abrupt onset, severe intensity or a ripping/ tearing sensation.

Clinical manifestations of ATAAD indicating perfusion deficit (pulse deficit, systolic blood pressure differential, focal neurological deficit plus pain), new aortic insufficiency murmur with pain, hypotension or shock are subsumed in the third aspect of the ADD-RS.

\section{Potential for improvement in pre-hospital EMS settings}

In patients with ATAAD, the quality of initial history is associated with the accuracy of the clinical diagnosis (9). In a retrospective study, Rosman et al. [1998] found that despite better knowledge, less than half of the patients with ATAAD had been asked the three essential questions about quality, radiation and intensity at the onset of pain. This is despite the ADD-RS being highly recommended by the Acute Cardiovascular Care Association of the European Society of Cardiology which clearly recommends checking for a bilateral systolic blood pressure differential and auscultating the heart for new aortic insufficiency murmur $(1,8)$.

In the unpublished data, quality estimations show less than 20 percent of the patients with ATAAD that were admitted to the DHZB have documented bilateral blood pressure measurements, and there are no documented auscultation results.

Rosman et al. [1998] suspected if physicians were confronted with highly stressed emergency patients, they would skip essential parts of the clinical evaluation and turn to technology-based diagnostic methods to speed up the diagnostic process.

A simple procedure is to perform a thoracic ultrasound scan. The focused heart ultrasound (FOCUS) is a straightforward technical procedure that can be quickly learned by doctors in the rescue centers.

\section{Bilateral systolic blood pressure differential}

A difference in in-arm blood pressure measurements is indicative for ATAAD, but it is not specific $(5,8,10)$. It can also occur in congenital heart disease, peripheral vascular disease and unilateral neuromuscular abnormalities.

A recent controlled study associated a bilateral systolic blood pressure differential of more than $20 \mathrm{mmHg}$ with ATAAD (10). Given the limited diagnostic options available in pre-hospital settings, a distinct differential might be the cue required to take an ATAAD into consideration.

\section{New murmur of aortic insufficiency}

The presence or absence of a new diastolic murmur of aortic regurgitation as standalone diagnostic criteria is not helpful, but in conjunction with pain, it is a high-risk factor as described in the ADD-RS $(3,8)$.

In an emergency setting (pre-hospital or otherwise) your chances of determining a murmur are minimal. Factors that may impede attempting such are the limited diagnostic tools, and the health care professional's level of experience. Despite this the attempt to diagnose this symptom in ATAAD patients should not be overlooked.

\section{The SMART approach}

To improve the response times in suspected ATAAD patients, we must use diagnostic options that are easily accessible in pre-hospital settings to gain diagnostic evidence (5).

Due to chest pain being the chief complaint, almost 40 percent of the patients with ATAAD are initially misdiagnosed with acute coronary syndrome (ACS) (5). The misinterpretation of ATAAD as ACS can result in an inadvertent emergency anticoagulation which will more than likely be detrimental to the patient $(5,7)$.

Evaluating a bilateral systolic blood pressure differential, or auscultating the heart are easy diagnostic tools that can be quickly used with every patient without adding any further stress to the patient or delaying any further processes.

For a better adherence to the ADD-RS, we propose introducing the 'SMART' concept (Figure 1) as a cue to remember what is widely known, but what is easily overlooked when confronted with a highly stressed emergency patient.

\section{The bottom line}

ATAAD is a dynamic process, starting with a broad variety 

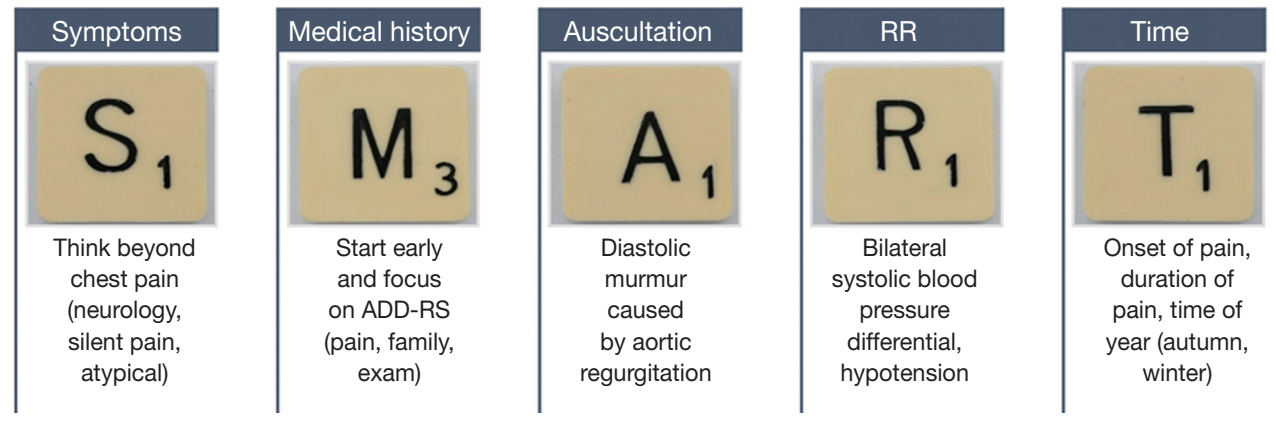

Figure 1 The SMART approach.

of symptoms that has the potential to lead to complications often requiring life support $(1,2)$.

Structured examination techniques, early communication of suspected ATAAD and the targeted use of quick and easy to use technical equipment for the focused detection of pathologies could significantly speed up the diagnosis of many patients (5-8).

\section{Acknowledgements}

We would like to thank Tegan Alexandra Steen of Melbourne, Australia for her assistance in linguistics on this manuscript.

\section{Footnote}

Conflicts of Interest: The authors have no conflicts of interest to declare.

\section{References}

1. Begui F, Castren M, Brunetti ND, et al. Pre-hospital management of patients with chest pain and/or dyspnoea of cardiac origin. A position paper of the Acute Cardiovascular Care Association (ACCA) of the ESC.

Eur Heart J Acute Cardiovasc Care 2015. [Epub ahead of print].

2. Schoenhagen P, Roselli EE, Harris CM, et al. Online network of subspecialty aortic disease experts: Impact of "cloud" technology on management of aortic emergencies. J Thorac Cardiovasc Surg 2016;152:39-42.

3. von Kodolitsch Y, Schwartz AG, Nienaber CA. Die klinische Diagnose akuter Aortendissektionen. Z Kardiol 2001;90:339-47.
4. Fleischmann D, Mitchell RS, Miller DC. Acute aortic syndromes: new insights from electrocardiographically gated computed tomography. Semin Thorac Cardiovasc Surg 2008;20:340-7.

5. Buschmann CT, Pappert T, Kurz SD. Die akute Aortendissektion Stanford Typ A: Kritische Schritte in der prä- und innerklinischen Notfallmedizin. In: Kuckelt W, Tonner PH, editors. Jahrbuch Intensivmedizin 2017. Pabst Science Publishers, Lengerich, 2017;109-17.

6. Kurz SD, Falk V, Kempfert J, et al. Insight into the incidence of acute aortic dissection in the German region of Berlin and Brandenburg. Int J Cardiol 2017;241:326-9.

7. Kurz S, Gieb M, Kuppe H, et al. Die akute Typ-ADissektion nach Stanford - Fallstricke und Implikationen für die notfallmedizinische Praxis. Notarzt 2017;33:20-4.

8. Rogers AM, Herman LK, Booher AM, et al. Sensitivity of the Aortic Dissection Detection Score, a Novel GuidelineBased Tool for Identification of Acute Aortic Dissection at Initial Presentation. Results from the International Registry of Acute Aortic Dissection. Circulation 2011;123:2213-8.

9. Rosman HS, Patel S, Borzak S, et al. Quality of history taking in patients with aortic dissection. Chest 1998;114:793-5.

10. Um SW, Ohle R, Perry JJ. Bilateral blood pressure differential as a clinical marker for acute aortic dissection in the emergency department. Emerg Med J 2018;35:556-8.

Cite this article as: Zschaler S, Schmidt G, Kurz SD. Aortentelefon: the Berlin project aiming for shorter response times and sharper diagnostic accuracy in acute type A aortic dissection. Cardiovasc Diagn Ther 2018;8(6):811-813. doi: 10.21037/cdt.2018.09.19 